\title{
Lamivudine monotherapy in children and adolescents: The devil is in the detail
}

\author{
L Fairlie, ${ }^{1}$ MB ChB, FCPaed (SA), MMed; J Bernheimer, ${ }^{2}$ MD, MSc, DTM\&H; N Sipambo, ${ }^{3}$ MB BCh, FCPaed (SA); C Fick, ${ }^{1,4}$ MB BCh \\ L Kuhn, ${ }^{5} \mathrm{PhD}$ \\ ${ }^{1}$ Wits Reproductive Health and HIV Institute, Faculty of Health Sciences, University of the Witwatersrand, Johannesburg, South Africa \\ ${ }^{2}$ Médecins Sans Frontières (Doctors Without Borders), Khayelitsha, South Africa \\ ${ }^{3}$ Chris Hani Baragwanath Academic Hospital, Department of Paediatrics and Child Health, Faculty of Health Sciences, \\ University of the Witwatersrand, Johannesburg, South Africa \\ ${ }^{4}$ Department of Paediatrics and Child Health, Faculty of Health Sciences, University of the Witwatersrand, Johannesburg, South Africa \\ ${ }^{5}$ Gertrude H Sergievsky Center, College of Physicians and Surgeons; and Department of Epidemiology, Mailman School of Public Health, \\ Columbia University, New York, NY, USA
}

Corresponding author: L Fairlie (lfairlie@wrhi.ac.za)

\begin{abstract}
Although expanded access to antiretroviral therapy (ART), and starting lifelong ART as soon as possible after diagnosis of HIV, have dramatically improved survival and reduced morbidity in HIV-infected children and adolescents, $\sim 20 \%$ of children will develop virological failure (VF). Children and adolescents may be at higher risk of VF and drug resistance for a number of reasons, including prevention of mother-to-child exposure, reliance on a caregiver to administer ART, poor palatability of paediatric drugs, tuberculosis/HIV co-treatment in protease inhibitor (PI) (mainly lopinavir/ritonavir)-based regimens, and adolescence being associated with poor adherence. In children with VF, if adherence issues are addressed and re-suppression is not achieved, a switch to second- or third-line drugs may be indicated, which is the gold standard in management. However, in the face of ongoing adherence challenges, with potential accumulation of resistance mutations, limited treatment options due to extensive resistance and limited approved paediatric formulations, other strategies have been used. These include continuing a failing PI regimen, switching to a holding regimen (one or more nucleoside reverse transcriptase inhibitors) or discontinuing ART. Lamivudine monotherapy is a common choice when holding regimens are used, on the premise that the lamivudineassociated $\mathrm{M} 184 \mathrm{~V}$ resistance mutation reduces viral replication and may maintain clinical and immunological stability compared with discontinuing treatment altogether. However, this strategy is generally associated with immunological, and in some cases clinical, decline after starting lamivudine monotherapy. We discuss the pros and cons of using this therapy in children. We also propose guidance for using lamivudine monotherapy, suggesting clinical and immunological criteria for its use. Close monitoring and adherence support are required with this approach. Given many new emerging ART drugs and strategies, lamivudine monotherapy should be administered temporarily, while efforts to improve adherence are implemented. It should not be considered a default option in children with VF.
\end{abstract}

S Afr Med J 2017;107(12):1055-1057. DOI:10.7196/SAMJ.2017.v107i12.12776

Success with prevention of mother-to-child transmission (PMTCT) has resulted in a substantial reduction in the number of HIV-infected children. ${ }^{[1]}$ Expanded access to antiretroviral therapy (ART), and recommendations to start lifelong ART as soon as possible after diagnosis, have dramatically improved survival and reduced morbidity in HIVinfected children and adolescents. ${ }^{[2]}$ Current HIV treatment guidelines for children recommend a protease inhibitor (PI)-based regimen (lopinavir/ ritonavir (LPV/r)) in those $<3$ years of age and a non-nucleoside reverse transcriptase inhibitor (NNRTI)-based regimen in those $>3$ years old, combined with 2 nucleoside reverse transcriptase inhibitors (NRTIs). ${ }^{[3,4]}$

In longitudinal follow-up studies, $\sim 20 \%$ of children develop virological failure (VF) 3 years after ART initiation. ${ }^{[5]}$ In these children, associated drug resistance mutations (RMs) may occur in up to $90 \%$. The most common mutations are associated with NNRTIs, lamivudine and thymidine analogues, with the lamivudine-associated mutation, M184V, occurring frequently. ${ }^{[6]}$ In children on first-line PI regimens, detected NNRTI RMs would have been selected by PMTCT.

In children, unique factors may result in poor adherence and/ or VF. These include exposure to nevirapine or efavirenz for PMTCT, with expected universal selection of NNRTI mutations in the exposed, and even modest rates of RMs in the unexposed (a reported rate of $24 \%$ in a PMTCT-unexposed population in South Africa (SA)). ${ }^{[7]}$ Children are also caregiver dependent, and thus completely reliant on an adult for ART administration. Any social or financial instability or change threatens the sustainability of their treatment. Furthermore, there is poor palatability of certain paediatric antiretrovirals, particularly LPV/r, and in some cases lack of availability of paediatric formulations, which requires adaptation of adult formulations. Tuberculosis (TB) co-treatment is common, raising complexities of drug-drug interactions between rifampicin and $\mathrm{LPV} / \mathrm{r}$, which is used as first-line treatment in children. Failure to boost LPV/r, ideally with the addition of ritonavir, or worse still, in early HIV/TB a switch to ritonavir as a single PI with rifampicinbased co-treatment (recommended by treatment guidelines at the time) has resulted in PI-associated RMs. ${ }^{[10,11]}$ Adolescence is also a challenging time for adherence. The abovementioned factors possibly conspire to increase the risks of VF and resistance in children and adolescents compared with their adult counterparts.

\section{Virological failure in children}

In children with VF, if issues related to poor adherence can be addressed, and re-suppression is not achieved while on the current 
regimen, a switch to a second- or third-line regimen may be indicated. Currently, children failing a first-line NNRTI-based regimen are switched to a PI-based regimen, which is fairly straightforward. More complicated is the scenario when children fail a first- or second-line PI-based regimen. Generally, PI-based regimens may allow lower adherence compared with an NNRTI-based regimen, as several mutations are required to confer resistance and rarely are selected, i.e. there is a high genetic barrier to resistance. The World Health Organization (WHO) recommends a switch from failing PI-based to NNRTI-based ART. ${ }^{[3]}$ However, this alternative has not been studied well and should be further investigated. Resistance testing may be helpful to guide the further management and to select drugs that can be included in the next regimen. The clinical use of resistance testing depends on which drugs were included in the initial regimen and the availability of other agents. These second- or third-line regimens following PI-based ART usually include integrase strand transfer inhibitors (INSTIs), a PI (older or new generation) and possibly an NNRTI (new generation). In SA, atazanavir is routinely available for children $>12$ years of age and darunavir on a special application basis for children $>3$ years old. Children able to access these options generally do well clinically and virologically on third-line regimens. ${ }^{[3,12-14]}$

Switching to a new susceptible regimen is the gold standard in managing children with VF. However, in the face of ongoing adherence challenges with potential accumulation of RMs, and limited treatment options due to extensive resistance and limited approved paediatric formulations, other strategies, such as continuing a failing PI regimen, switching to a holding regimen or discontinuing ART, have been used. ${ }^{[15,16]}$ In comparative studies in the USA and SA, switching to a definitive regimen has been shown to have preferable virological and immunological outcomes than when children remain on their failing regimen, switch to a holding regimen or discontinue ART. $^{[15,16]}$

\section{Holding regimens}

In cases where ongoing poor adherence is expected and there is concern regarding increased accumulation of RMs, or where few new drugs are available, strategies to 'buy time' while improving adherence have been employed. Holding regimens are defined as a switch to one or more NRTIs to provide some clinical and immunological benefit without the expectation of full viral suppression until adherence can be achieved and/or an alternative regimen can be obtained. Most commonly, lamivudine monotherapy is used as a holding regimen in children with known lamivudine resistance, on the premise that the lamivudine-associated M184V mutation reduces viral replication. ${ }^{[17]}$ While this may be an option in certain situations, it should be used with caution and not as a default regimen in children who fail ART. Few data exist on the safety and efficacy of this holding regimen. Studies generally show immunological and sometimes clinical deterioration when this strategy is used. Studies from Johannesburg, the Eastern Cape and the multicentre International epidemiology Databases to Evaluate AIDS (IeDEA) cohort, reported a $\leq 25 \%$ decline in CD4+ count compared with time of switch after 6 months of lamivudine monotherapy in up to $72 \%$ of children, while in another study $8 \%$ of children deteriorated to WHO stage 3 or 4 disease. ${ }^{[18-20]}$ The only randomised controlled study comparing lamivudine monotherapy with continuation of a failing PI-based regimen, IMPAACT P1094, enrolled 33 children and adolescents; it was discontinued owing to lack of accrual and delays in approval by ethics committees. ${ }^{[21]}$ This study reported a $>30 \%$ decline in CD4+ count in those receiving lamivudine monotherapy compared with those continuing a failing PI without clinical deterioration, leading to a recommendation by some guideline bodies that this strategy should not be routinely recommended. ${ }^{[21,22]}$ Using additional NRTIs does not appear to mitigate CD4+ count decline. A study from New York, where children failing a PI-based regimen continued with $\geq 2$ NRTIs, showed that at 48 weeks, there was a statistically significant decline in CD4+ count compared with baseline; however, there was no control group. ${ }^{[23]}$

An additional concern with holding regimens that allow continuing viraemia is the possibility of increased risks of neurocognitive decline in children. HIV-infected children, even those who receive ART, have significant neurocognitive delay compared with their HIVunexposed and HIV-exposed uninfected counterparts. ${ }^{[2]}$ In the context of planned treatment interruption in the Children with HIV Early Antiretroviral Therapy (CHER) trial, motor development was affected. ${ }^{[25]}$ Nevertheless, with close observation and re-initiation of ART, recovery was possible (B Laughton - personal communication). The Paediatric European Network for Treatment of AIDS 11 (PENTA 11) study found no neurocognitive deterioration with ART interruption. ${ }^{[26]}$ In adults, treatment interruption is discouraged, largely based on the Strategies for Management of Antiretroviral Therapy (SMART) study, which found poorer outcomes in those who discontinued ART than in those who continued while being suppressed, attributable to unchecked inflammation and increased risk of cardiovascular disease. ${ }^{[27]}$ In contrast, in children, no adverse clinical effects of short-term treatment interruptions have been observed. The reasons for halting the treatment interruption studies in children, such as in the OHP-03, have not been because of safety, but owing to low treatment interruption durability, i.e. futility of their intended purpose to lead to remission. ${ }^{[25,28,29]}$

One of the motivations for the use of a holding regimen is to allow children and adolescents to practise adherence to their ART regimen. This may be advantageous, but may also introduce new adherence challenges and misunderstandings. For instance, children may misinterpret lamivudine monotherapy as a safe and easy regimen and may prefer it to ART. This could make it difficult to switch back to a suppressive regimen when necessary, based on immunological or clinical criteria. Children who receive lamivudine monotherapy also require intense clinical and adherence follow-up, including at least 3-monthly CD4+ count monitoring. This may be problematic for children who have socioeconomic problems at the heart of their poor adherence, where increased visits are simply not affordable. Ongoing enhanced adherence support is essential in these children and their families to enable an eventual switch back to a definitive ART regimen. An additional factor is that lamivudine monotherapy administered by less experienced clinicians may result in children and adolescents only accessing suppressive ART after severe clinical and immunological deterioration. Therefore, construing this option as safe for children may be misleading for clinicians and caregivers and should not be a default option in all children who fail ART, especially those failing second-line regimens.

\section{Recommendations for lamivudine monotherapy in children}

There are, however, specific situations where it may be necessary to consider a holding regimen. We propose the following inclusion criteria for recommending lamivudine monotherapy or holding regimens:

- Clinically well child (growth stable along centiles, no current or recent clinical disease expected to recur or re verse remission with immunological deterioration, including most WHO stage 4 conditions, such as extrapulmonary $\mathrm{TB}$, cryptococcal disease, Mycobacterium avium complex, lymphoma, Kaposi sarcoma and HIV-associated neurological disease). Children with proven previous pulmonary TB should be excluded. 
- CD4+ count $>30 \%$ and $>500$ cells $/ \mu \mathrm{L}$ - to be checked 3-monthly.

- Able to attend the clinic for monthly follow-up.

- Able to conduct adherence counselling and support at each clinic visit.

- Child should be $>5$ years old, unless there are no other options for treatment.

- Success of lamivudine treatment should be measured by a switch to a definitive ART regimen.

New regimens for children/adolescents There are problems with constructing new regimens for children with extensive resistance, which include challenges with regard to dosing and safety data for new ARVs, TB co-treatment, cost and availability. However, it is anticipated that newer drugs, such as dolutegravir, with a reduced side-effect and improved resistance profile, will be available in the next few years. It is hoped that these drugs will reduce the need for regimen switches in children with VF. Other new INSTIs, NNRTIs and PIs are currently being evaluated in phase I/II studies, as are improved formulations for delivery, such as injectable ARVs, including cabotegravir and rilpivirine. Research is also being done on therapeutic HIV vaccines and neutralising antibodies as treatment adjuvants. Investigation of new drugs in children is often delayed compared with that in adolescents and adults; often these studies do not include formulations palatable and easy to administer in children. However, over recent years there has been substantial progress in drug development, with numerous studies evaluating new ART in newborns, children and adolescents, making it likely that in the near future improved variety and efficacy of therapeutic options will be available for children.

\section{Conclusion}

The management of $\mathrm{VF}$ in children is challenging. Improving adherence is the most important component. Key management points to consider are: minimising the accumulation of resistance mutations, ensuring that the child's clinical condition remains stable, preventing new opportunistic infections or reversing remission of latent infections, and optimising growth and neurocognitive and educational development. Alternative options to fully suppressive ART should be used only in special circumstances, with substantial support, rather than as a default option.

Acknowledgements. We acknowledge all the children and their families whom we have the privilege of caring for.

Author contributions. All authors were involved in conceiving the manuscript and developing its content. All authors reviewed the manuscript and approved the final version.

Funding. LF and CF are supported by the President's Emergency Plan for AIDS Relief (PEPFAR)/United States Agency for International Development (USAID) funding (ref. no. IU0IGH002094_01).

Conflicts of interest. None.

\footnotetext{
1. United States Agency for International Development. Get on the Fast-Track. Finding Solutions for Everyone at Every Stage of Life. Washington, DC: USAID, 2016.

2. Violari A, Cotton MF, Gibb DM, et al. Early antiretroviral therapy and mortality among HIV-infected infants. N Engl J Med 2008;359(21):2233-2244. https://doi.org/10.1056/NEJMoa080097
}

3. World Health Organization. Consolidated Guidelines on the Use of Antiretroviral Drugs for Treating and Preventing HIV Infection: Recommendations for a Public Health Approach. Geneva: WHO, 2013.

4. National Department of Health. National Consolidated Guidelines for the Prevention of Mother-toNational Department of Health. National Consolidated Guidelines for the Prevention of Mother-to-
Child Transmission of HIV (PMTCT) and the Management of HIV in Children, Adolescents and Adults. Pretoria: $\mathrm{NDoH}, 2015$.

Adults. Pretoria: NDoH, 2015.
5. Davies MA, Moultrie H, Eley B, et al. Virologic failure and second-line antiretroviral therapy in children in South Africa - the IeDEA southern Africa collaboration. J Acquir Immune Defic Syndr 2011;56(3):270-288. https://doi.org/10.1097/QAI.0b013e3182060610

6. Muri L, Gamell A, Ntamatungiro AJ, et al. Development of HIV drug resistance and therapeutic failure in children and adolescents in rural Tanzania: An emerging public health concern. AIDS 2017;31(1):61-70. https://doi.org/10.1097/qad.0000000000001273

7. Church JD, Mwatha A, Bagenda D, et al. In utero HIV infection is associated with an increased risk of nevirapine resistance in Ugandan infants who were exposed to perinatal single dose nevirapine. AIDS nevirapine resistance in Ugandan infants who were exposed to perinatal single dose
Res Human Retrovirus 2009;25(7):673-677. https://doi.org/10.1089/aid.2009.0003

8. Martinson NA, Morris L, Gray G, et al. Selection and persistence of viral resistance in HIV-infected children after exposure to single-dose nevirapine. J Acquir Immune Defic Syndr 2007;44(2):148-153. https://doi.org/10.1097/QAI.0b013e31802b920e

9. Kuhn L, Hunt G, Technau KG, et al. Drug resistance among newly diagnosed HIV-infected children in the era of more efficacious antiretroviral prophylaxis. AIDS 2014;28(11):1673-1678. https://doi org/10.1097/qad.0000000000000261

10. Rossouw TM, Feucht UD, Melikian G, et al. Factors associated with the development of drug resistance mutations in HIV-1 infected children failing protease inhibitor-based antiretroviral therapy in South Africa. PLoS ONE 2015;10(7):e0133452. https://doi.org/10.1371/journal.pone.0133452

11. Van Zyl GU, van der Merwe L, Claassen M, et al. Protease inhibitor resistance in South African an Zyl GU, van der Merwe L, Claassen M, et al. Protease inhibitor resistance in South African
children with virologic failure. Pediatr Infect Dis J 2009;28(12):1125-1127. https://doi.org/10.1097/ INF.0b013e3181af829d

12. Kindra G, Sipambo N, Moultrie H, Fairlie L. Outcomes in treatment with darunavir/ritonavir in ARTexperienced paediatric patients. S Afr Med J 2015;105(5):330-331. https://doi.org/10.7196/samj.9211

13. Dehority W, Abadi J, Wiznia A, Viani RM. Use of integrase inhibitors in HIV-infected children and adolescents. Drugs 2015;75(13):1483-1497. https://doi.org/10.1007/s40265-015-0446-2

14. Lazarus E, Nicol S, Frigati L, et al. Second- and third-line antiretroviral therapy for children and adolescents: A scoping review. Pediatr Infect Dis J 2017;36(5):492-499. https://doi.org/10.1097/inf.0000000000001481

15. Fairlie L, Karalius B, Patel K, et al. CD4+ and viral load outcomes of antiretroviral therapy switch strategies after virologic failure of combination antiretroviral therapy in perinatally HIV-infected youth strategies after virologic failure of combination antiretroviral therapy in perinatally HIV-infected youth
in the United States. AIDS 2015;29(16):2109-2119. https://doi.org/10.1097/qad.0000000000000809

16. Patten G, Schomaker M, Davies M-A, et al., eds. What Should We do when HIV-positive Children Patten G, Schomaker M, Davies M-A, et al., eds. What Should We do when HIV-positive Children
Fail First-line Combination Anti-retroviral Therapy? A Comparison of 4 ART Management Strategies. Paris: International AIDS Society, 2017.

17. Gallant JE, Gerondelis PZ, Wainberg MA, et al. Nucleoside and nucleotide analogue reverse transcriptase inhibitors: A clinical review of antiretroviral resistance. Antiviral Ther 2003;8(6):489-506. 8. Linder V, Goldswain C, Adler $\mathrm{H}$, et al. Lamivudine monotherapy: Experience of medium-term outcomes in HIV-infected children unable to adhere to triple therapy. Pediatr Infect Dis 2016;35(7):e199-e205. https://doi.org/10.1097/inf.0000000000001156

19. Patten GEM, Bernheimer J, Cox V, et al. Outcomes in HIV-positive children on lamivudine monotherapy as a holding regimen in the IEDEA southern African cohorts. 8th International monotherapy as a holding regimen in the leDEA southern African cohorts. 8th Internationa Workshop on HIV Pediatrics, 15 - 16 July 2016, Durban, South Africa. http://www.
com/event/previous/hiv-pediatrics-workshop-2016/ (accessed 6 November 2017).

20. Lazarus EM, Otwombe K, Fairlie L, et al. Lamivudine monotherapy as a holding strategy in HIV-infected children in South Africa. J AIDS Clin Res 2013;4(10). https://doi.org/10.4172/2155-6113.1000246

21. Agwu AL, Warshaw M, McFarland E, et al. Decline in CD4 T lymphocytes with monotherapy bridging strategy for non-adherent adolescents living with HIV infection: Results of the IMPAACT P1094 randomized trial. PLoS ONE 2017;12(6):e0178075. https://doi.org/10.1371/journal.pone.0178075.

22. Panel on Antiretroviral Therapy and Medical Management of Children Living with HIV. Guidelines fo the use of antiretroviral agents in pediatric HIV infection. 2017. https:/aidsinfo.nih.gov/guidelines/ $\mathrm{html} / 2 /$ pediatric-arv/0 (accessed 6 November 2017).

23. Abadi J, Sprecher E, Rosenberg MG, et al. Partial treatment interruption of protease inhibitor-based Abadi J, Sprecher E, Rosenberg MG, et al. Partial treatment interruption of protease inhibitor-based
highly active antiretroviral therapy regimens in HIV-infected children. J Acquir Immune Defic Syndr highly active antiretroviral therapy regimens in HIV-infected children. J A
2006;41(3):298-303. https://doi.org/10.1097/01.qai.0000197078.41150.0d

24. Boivin M, Chernoff M, Zimmer B, et al. Neuropsychological performance in African children with HIV enrolled in a multi-site anti-retroviral clinical trial is poorer than non-infected children a those study sites. 8th International Workshop on HIV Pediatrics, 15 - 16 July 2016, Durban, South Africa. http://www.virology-education.com/event/previous/hiv-pediatrics-workshop-2016/ (accessed 6 November 2017).

25. Cotton MF, Violari A, Otwombe K, et al. Early time-limited antiretroviral therapy versus deferred therapy in South African infants infected with HIV: Results from the children with HIV early antiretroviral (CHER) randomised trial. Lancet 2013;382(9904):1555-1563. https://doi.org/10.1016/ antiretroviral (CHER)

26. Ananworanich J, Melvin D, Amador JT, et al. Neurocognition and quality of life after reinitiating antiretroviral therapy in children randomized to planned treatment interruption. AIDS 2016;30(7):10751081. https://doi.org/10.1097/qad.0000000000001011

27. El-Sadr WM, Lundgren J, Neaton JD, et al. CD4+ count-guided interruption of antiretroviral treatment. N Engl J Med 2006;355(22):2283-2296. https://doi.org/10.1056/NEJMoa062360

28. Wamalwa D, Benki-Nugent S, Langat A, et al. Treatment interruption after 2-year antiretroviral treatment initiated during acute/early HIV in infancy. AIDS 2016;30(15):2303-2313. https://doi.org/10.1097/ qad.0000000000001158

29. Klein N, Sefe D, Mosconi I, et al. The immunological and virological consequences of planned treatment interruptions in children with HIV infection. PLoS ONE 2013;8(10):e76582. https://doi. org/10.1371/journal.pone. 0076582

Accepted 28 September 2017. 